\title{
Promoting Motor Development and Future Motor Performance Optimization through Constraints and Affordances Manipulation
}

\author{
Rui Matos ${ }^{1,2}$ \\ ${ }^{1}$ CIEQV (Life Quality Research Center) - Polytechnic Institute of Santarém and Polytechnic Institute of Leiria, Leiria, Portugal \\ ${ }^{2}$ ESECS (School of Education and Social Sciences) - Polytechnic Institute of Leiria, Leiria, Portugal \\ Email: rui.matos@ipleiria.pt
}

How to cite this paper: Matos, R. (2017) Promoting Motor Development and Future Motor Performance Optimization through Constraints and Affordances Manipulation. Open Access Library Journal, 4: e4048. https://doi.org/10.4236/oalib.1104048

Received: October 20, 2017

Accepted: December 4, 2017

Published: December 7, 2017

Copyright $\odot 2017$ by author and Open Access Library Inc.

This work is licensed under the Creative Commons Attribution International License (CC BY 4.0).

http://creativecommons.org/licenses/by/4.0/

\begin{abstract}
People who work with children should be able to make an appropriate environment analysis where children move. By doing this, they could positively interfere and modify constraints, so that the detection (and creation) of affordances for action could occur easily. Taking this for granted, these children could, then, engage into motor problems resolution, looking for the best solutions for each dilemma that they are confronted with. In this paper, we reflect about educators, parents and coaches role on the promotion of children' motor development and, therefore, in the future motor performance optimization through constraints and affordances manipulation, with examples that stress and point out the importance of this pedagogic, methodologic and training procedure.
\end{abstract}

\section{Subject Areas}

Education, Sports Science

\section{Keywords}

Motor Development, Motor Performance Optimization, Constraints Manipulation, Affordances, Ecological Dynamics

\section{Introduction}

According to a development ecological perspective, constraints, which are seen as factors that have the potential to influence behavioural changes, shape or guide systems' self-organization, being a shoal of fishes moving in an orderly manner or a child dealing with a new motor task. Constraints relate with degrees of freedom [1] on the performance of motor tasks, being of special importance 
the notion that individuals and systems in general have the capacity to self-modify and adjust to changes that surround them [2] [3].

Thus, and according to Newell [4], the fact that we have the weight, the height, the strength, and so on, that we have, the rules, on sports, that we are compelled to follow, or the fact that the wind is blowing sideways on javelin throwing, lead us to modify our actions to adjust to those conditions, that is to say, actions become restrained by them. The same occurs when, in soccer or in another team sport, we train in a smaller pitch, keeping the same number of players, forcing them to think and act quicker-less space, less time. Looking to the other face of this coin, Gibson [5] introduced the term affordance, referring to what a certain environment permits a certain actor to perform. As an example, a very thick layer of ice affords people to walk on it but, being thin, it will only afford us to crawl on it or, at the limit, not to pass (no affordance at all, in fact). It's worth to stress that, using this example, we should not say that the ice layer does not afford to walk but, rather, does not afford me or some of us to walk, since, of course, someone less heavy could, probably, do it.

Another important aspect is that, despite many affordances can be easily detectable even for who does not have much experience in performing related tasks, it will be up to educators and teachers, especially those who work with children of lower age, but not only, to play an important role. In fact, every physical education teacher or professional coach idealize and design tasks where they play with constraints which get manipulated, affordances that children understand and motor solutions that they find more or less directly. In future sports practice, this will be very helpful, since sports, usually, require players to engage into problems resolution, situation that will, most probably, be well solved if they got acquainted, since early development, dealing with changing environments and direct perception of multimodal stimuli.

Let's pick ramps as an example. In abstract, they allow people to go down standing, sitting, rolling, etc. With the various attempts that children perform, they get to know the way that most fits their motor development stage, that is, despite the fact that, in the first few trials, some try to descend standing, by falling, they, before long, realize that the best way (more safe and effective) to overcome that obstacle will be to go down sitting, dragging, bending their knees (in order to lower gravity centre) or sliding like in a playground slide. When, finally, this understanding becomes stable, we might say, according to theories mentioned before, that the child learned how to act in that particular situation (attending multiple factors: slope of the ramp, smoothness of the surface, children ability to balance, etc.). This change in the category of action used by them (from standing to sitting) will, in practice, constitute their learning. Interestingly, later, its development and accumulated experience will lead a return, now appropriately, to the use of the category of action that, at first, was dysfunctional: to descend standing in an upright position. Norman [6], applying the concept of affordance to the world of design, points out that, in his profession, people care a lot more about what the user perceives than what is true. What the designer ca- 
res about is if the person who will use the product that he conceived understands that a certain action is possible. Thus, a given object intended to be used in a certain way (constraint) should highlight features, the most immediate and intuitive as possible, that induce the desired way of use (affordance). This will not be the case of a door that only opens in a given direction but, coupled, has a handle that allows it to be pulled or pushed. In this case, if the door had no handles, it would immediately be perceived that, at least, it could not be pulled, given that it would fail to provide the user with any affordance for that particular action.

Interestingly, in future sports practice, especially on those where two players or teams oppose each other, counter-information will be of great efficacy. In most of the cases, this counter-information will be based, precisely, on sending a wrong message to the opponents, leading them to think, for instance, that there is an affordance for their passage on a certain part of the pitch when, in fact, everything is prepared to, when they attempt that passage, close the path. This is the case of the information a goalkeeper gives to the striker, on a penalty-kick, when he moves himself to the right place of the goal. Striker, if not prepared, can be induced to kick the ball to the left side of the goalkeeper when, in fact, that is precisely what goalkeeper wants, being ready to make an explosive lower limbs muscle contraction that will lead him to that side. As can be easily understood, in sport, as important as becoming able to detect affordances and overcome and adjust to constraints, is being able to constraint opponents' actions, forcing them to perform with less chances of success and using fake affordances.

Returning to educators (in general) and their role in this game of constraints and affordances, we believe it is essential that they be able to handle these constraints and to highlight affordances, so that children, by themselves, are able to find the best solutions. The great goal is adaptive behaviour to emerge from the dynamic interaction between the individual and his involvement [7]. Since functional coordinative solutions can't be completely predicted, due to its construction as a function of the ecological constraints of each real motor situation, it will be up to Physical Education teachers and other staff who deal with children motor issues to create situations in which it is possible to establish a solid connection between perceptions and actions. More than to inculcate the use of rigid motor solutions, it will be important that children learn to modulate their behaviour in order to achieve a consistent performance [8].

It is interesting to note that small changes can lead to new affordances or, at least, their detection, giving rise, in this way, to new possibilities of action [9]. It can be up to teachers and coaches to help to identify and use these affordances, aiming the achievement of goals at a performative level. This help, we believe, becomes especially important in periods where there are significant changes in body dimensions and/or possibilities of action of subjects [10], as are the cases of puberty growth spurt and relatively low ages. It becomes especially important that these agents can devise, draw and manipulate tasks and/or environments so that constraints, namely at an informative level, can be more easily detectable 
and, this way, facilitate the finding of functional coordinative solutions. For example, we can mention a (physical) constraint that, in some playgrounds, can be observed in some slides, aiming at the assumption of a more secure behaviour: the existence of an horizontal bar, at the access to the platform from which to begin the descent, implies, physically, no allowance to the child to go down standing/on foot, being forced to take a lower posture, hopefully seated. Another example of situations created so that involvement becomes more user friendly is the painting of a strip of contrasting colour on the edge of walkways and/or stairs, aiming to highlight the consequent gap. In this case, more than a constraint, as shown in the previous example, we are dealing with an attempt to facilitate the detection of an affordance. To be even more precise, the aim is, in fact, that people detect more easily the non-affordance if keeping the form of displacement that they were using (amplitude of gait, speed of displacement, etc.), which could originate a fall. Therefore, and given that security depends, in a large measure, of the individual possibilities of action and ability to perceive what is or is not possible in certain circumstances and environments [11], it becomes, at least at this level (security in physical activities), important to adapt the environments and the tasks to perceptive (and motor) capabilities of children, which are, as mentioned above, in constant evolution.

However, and despite being possible, early on, to verify that children are able to detect the affordances for large part of the actions in which they are involved, there are, as pointed out by Barreiros [11], important differences between children and adults in the choice of categories of action and in the transition between them. According to the author just referred to, it will be precisely in the areas of uncertainty that may arise the biggest problems. Indeed, when, for example, a wall is clearly too high to jump to the ground from it, or so low that it does not raise any fears to run the action mentioned above, children and adults assume similar behaviours. However, when the height of that wall is at a borderline situation, mistakes, bad decisions and ineffective actions may arise (particularly dangerous in cases of overestimation of capabilities). A broader view of security should, therefore, include a strategy of provision of equipment emerging from children (anthropometric and functional) characteristics, aiming the reach of affordances [12]. In addition, and as already mentioned before, there should exist investment on a perceptive facilitation (not only visual but also tactile, kinaesthetic and auditory), taking into account issues such as figure-background, roughness/smoothness of surfaces (better relief perception and accentuation of dangerous slopes, etc.).

\section{Methods}

First of all, this should not be considered a traditional, nor even a pilot-study experimental approach. It is meant, primarily, to illustrate previous reflection. Keeping this in mind, we used two situations. One (Figure 1), spontaneous, emerging from a child's play, and another (Figure 2), deliberately created to promote the manifestation of different behaviours. 

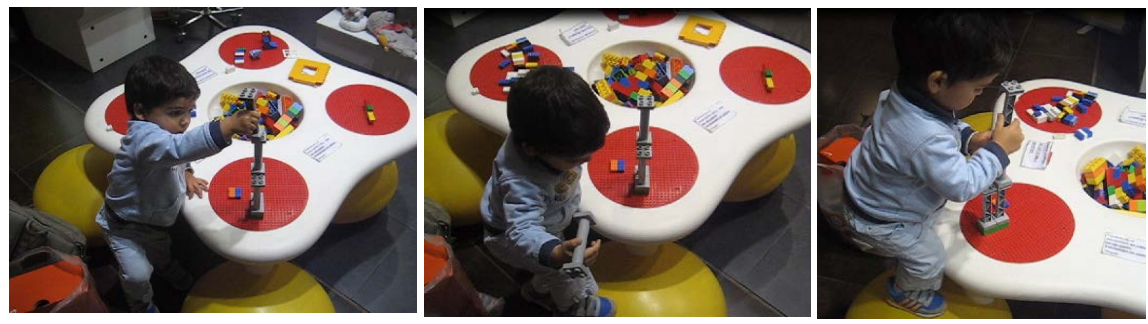

Figure 1. Having found (figure in the left) that, from the position in which she finds herself, the child can't put the piece on the top of the other, she detects the affordance that the base of the table gives her (Middle figure), and, having climbed, she places the piece successfully (figure on the right).
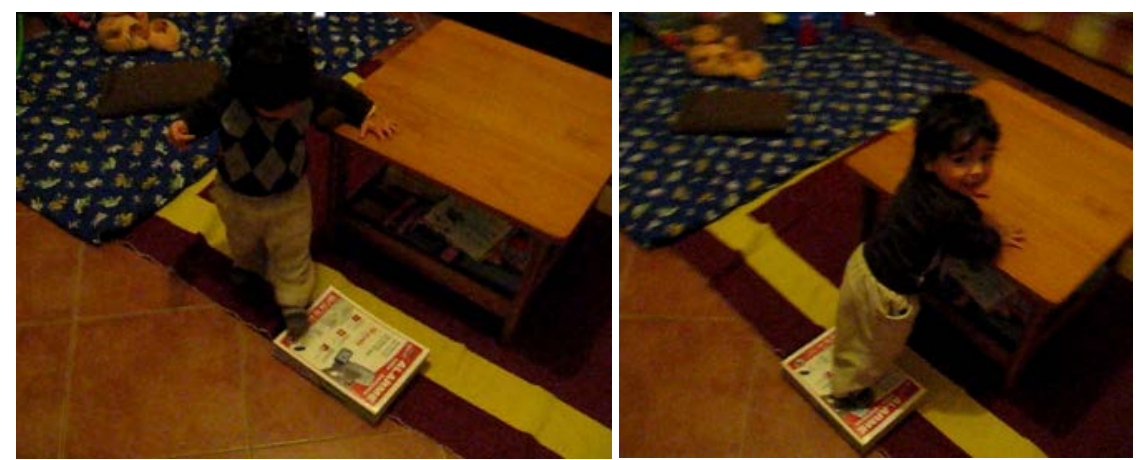

(a)
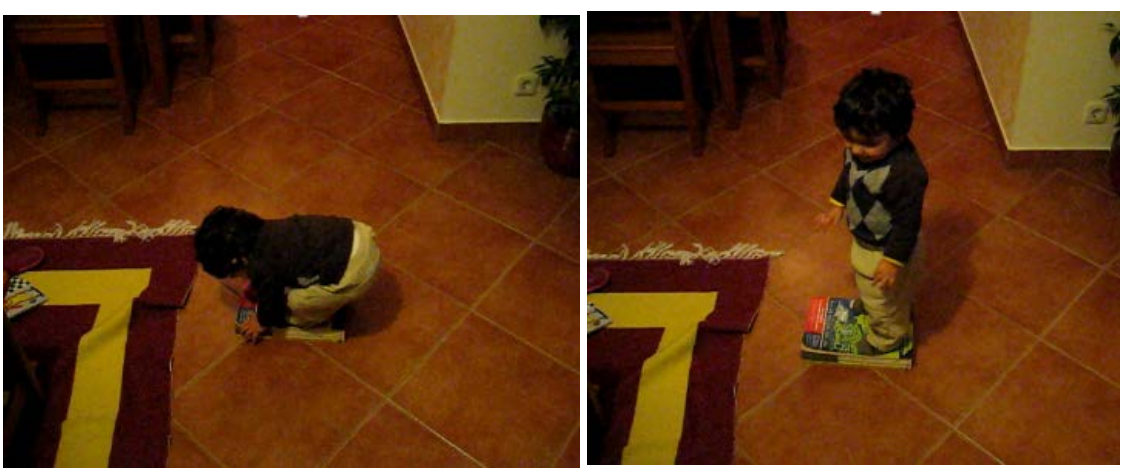

(b)

Figure 2. (a) The presence of a table offers an affordance for a stand passage up to the object in the floor; (b) By the contrary, the absence of a supporting object induces a different (curled) approach for passing up to the object in the floor.

Figure 1 and Figure 2 illustrate how objects (or their absence) that surround kids, at relatively low ages, can influence (and really influence) the way they move and behave in space (see [13], about affordances for development that can/should be given in the homemade and family involvement).

\section{Results}

As can be seen, this 17 months' boy clearly adapted his behavior and categories of action to the constraints he found, being them natural (Figure 1) or artificial, i.e., adapted by an adult/educator. These examples show very clearly, though no 
conclusions can/should be taken from one single case, that, from very early ages, children seem to be able to detect affordances on the environment that allow them to behave differently, according to constraints' influence and determinism.

\section{Conclusions}

Having in mind the main issue of our reflection, we think it is reasonable to say that educators, parents, coaches and all those who, in one way or another, have responsibilities in the (motor) development of children, should be very attentive and available to detect, handle and make constraints and affordances more detectable, in order to help children to find the best functional coordinative solutions, educating and training them to lead their own development in future.

It's worth to remark that, whether or not we decide to act, not acting is, already, a way of influencing, positively or negatively, the motor development of children. Sometimes, of course, it's wise to let them play with no adult interference, knowing that, anyway, constraints and affordances will always be there. However, knowing the importance constraints and affordances have on their development, it would represent a reckless conduct not no manipulate environment and tasks features so that they represent a more powerful, challenging, and richer stimulus to children.

As it can be easily understood, in sport, as important as becoming able to detect affordances and overcome and adjust to constraints is being able to constraint opponents' actions, forcing them to perform with less chances of success and trusting on fake affordances. This is something that should begin being done in early years. Children, young boys and girls and, later on, adolescents and adults, all will benefit from being exposed to different stimuli and difficulty levels, facilitating their self-knowing, their capabilities of action and, therefore, promoting a better adjustment on sports practice, where, usually, we have very fast changing conditions, forcing players to quick regulations and decision taking.

Following this reflection article, empirical work should be done to strength the knowledge of how children adapt their motor behavior when constraints are manipulated under certain and different conditions.

\section{Acknowledgements}

This work is financed by national funds through FCT-Fundação para a Ciência e a Tecnologia, I.P., within the framework of the project UID/CED/04748/2016.

\section{References}

[1] Bernstein, N.A. (1967) The Coordination and Regulation of Movements. Pergamon Press, Oxford.

[2] Smith, L.B. and Thelen, E. (1993) A Dynamical Systems Approach to Development: Applications. The MIT Press, Cambridge.

[3] Thelen, E. and Smith, L.B. (1994) A Dynamical Systems Approach to the Development of Cognition and Action. The MIT Press, Cambridge. 
[4] Newell, K.M (1986) Constraints on the Development of Coordination. In Wade M.G. and Whiting, H.T.A., Eds., Motor Development in Children Aspects of Coordination and Control, Martinus Nijhoff, Leiden, 341-360. https://doi.org/10.1007/978-94-009-4460-2_19

[5] Gibson, J.J. (1979) The Ecological Approach to Visual Perception. Houghton Mifflin, Boston.

[6] Norman, D.A. (1988) The Psychology of Everyday Things. Basic Books, New York.

[7] Hristovski, R., Davids, K., Araújo, D. and Passos, P. (2011) Constraints-Induced Emergence of Functional Novelty in Complex Neurobiological Systems: A Basis for Creativity in Sport. Nonlinear Dynamics, Psychology and the Life Sciences, 15, 175-206.

[8] Araújo, D. and Davids, K. (2011) What Exactly Is Acquired during Skill Acquisition? Journal of Consciousness Studies, 18, 7-23.

[9] Turvey, M.T. and Shaw, R. (1999) Ecological Foundations of Cognition I: Symmetry and Specificity of Animal-Environment Systems. Journal of Consciousness Studies, 6, 95-110.

[10] Renshaw, I. (2010) A Constraints-Led Approach to Talent Development in Cricket. In Kidman, L. and Lombardo, B., Eds., Athlete-Centred Coaching, NZ: Innovative, Christchurch, 151-172.

[11] Barreiros, J. (2006) Aprendizagem e percepção de risco. Congresso Espaços de Jogo e Recreio: Lei, Inovação e Boas Práticas, organizado pela APSI. Lisboa, Junho de 2006.

[12] Barreiros, J. and Silva, P. (1995) Hand Size and Grasping in Infants. In Bardy, B.G., Bootsma, R. and Guiard, Y., Eds., VIII th International Conference on Perception and Action, Lawrence Erlbaum Associates, Abingdon.

[13] Rodrigues, L., Saraiva, L. and Gabbard, C. (2005) Development and Structural Validation of an Inventory for Assessing Affordances in the Home Environment for Motor Development. Research Quarterly for Exercise and Sport, 76, 140-148. https://doi.org/10.1080/02701367.2005.10599276 